\title{
A new approach to alarm management: mitigating failure-prone systems
}

\author{
Adalberto Torres ${ }^{1}$, David E Milov ${ }^{1}$, Daniela Melendez ${ }^{1}$, J oseph Negron ${ }^{1}$, J ohn J Zhao ${ }^{2}$, Stephen T \\ Lawless $^{1,2}$ \\ 1. Nemours Children's Hospital, Orlando, FL, United States. 2. Nemours/Alfred I. duPont Hospital for Children, Wilmington, \\ DE, United States.
}

Correspondence: Adalberto Torres. Address: 13535 Nemours Parkway, Orlando, FL 32827, United States. E-mail: al.torres@nemours.org

Received: June 3, 2014

Accepted: August 22, $2014 \quad$ Online Published: October 9, 2014

DOI : $10.5430 /$ jha.v3n6p79

URL: http://dx.doi.org/10.5430/jha.v3n6p79

\begin{abstract}
Alarm management that effectively reduces alarm fatigue and improves patient safety has yet to be convincingly demonstrated. The leaders of our newly constructed children's hospital envisioned and created a hospital department dedicated to tackling this daunting task. The Clinical Logistics Center (CLC) is the hospital's hub where all of its monitoring technology is integrated and tracked twenty-four hours a day, seven days a week by trained paramedics. Redundancy has been added to the alarm management process through automatic escalation of alarms from bedside staff to CLC staff in a timely manner. The paramedic alerting the bedside staff to true alarms based on good signal quality and confirmed by direct visual confirmation of the patient through bedside cameras distinguishes true alarms from nuisance/false alarms in real time. Communication between CLC and bedside staff occurs primarily via smartphone texts to avoid disruption of clinical activities. The paramedics also continuously monitor physiologic variables for early indicators of clinical deterioration, which leads to early interventions through mechanisms such as rapid response team activation. Hands-free voice communication via room intercoms facilitates CLC logistical support of the bedside staff during acute clinical crises/resuscitations. Standard work is maintained through protocol-driven process steps and serial training of both bedside and CLC staff. This innovative approach to prioritize alarms for the bedside staff is a promising solution to improving alarm management.
\end{abstract}

\section{Key words}

Clinical logistics, Physiologic monitoring, Patient safety

\section{I ntroduction}

Advances in technology and an increasing acuity of hospitalized patients have led to widespread deployment of bedside physiologic monitors (BPMs). The BPMs are used to monitor for an acute change in a patient's clinical status or to suggest more subtle trends in status that inform the health care team. In response to changes, BPMs may emit a characteristic sound (alarm). Alternatively, a trained observer may note the display of abnormal waveforms or other visual display. The additional work burden created by BPMs has fallen primarily on the nursing staff members of the health care team. Current monitoring methods are real time and non-distributed and yield best effort, as opposed to fail-safe response. 
Systems theory holds that systems failure is most common with small stepwise deviation rather than a massive single-step failure. Moreover, there is a linear relationship between medical error and the number of tasks assigned. In practical terms, monitored patients add several tasks to the care team even when a patient is improving. In addition, BPMs are not routinely integrated with the electronic medical record (EMR); thus, BPMs create a documentation burden without benefit of computer-assisted decision support. Since the positive predictive value of a given alarm is low and the rate of false alarms is high, alarm desensitization becomes common, understandable, and an increasingly common risk to patients ${ }^{[1]}$. This failure to appreciate, acknowledge, or respond to alarms has been termed alarm fatigue. The current practice in alarm response is dependent on a single person. Outcomes for a system with endemic alarm fatigue that depends on a single observer response are far from fail-safe. Best effort service level response to alarms is not good enough to keep patients safe.

Despite the advances in medical technology over the last 20 years, the proportion of patient alarms that are clinically relevant remains extremely low at approximately $5 \%{ }^{[1,2]}$. Alarm fatigue, a result of the disproportionate amount of nuisance/false alarms bombarding hospital staff, was identified as the primary cause for 80 hospital deaths reported to the Joint Commission from 2009 to $2012^{[3]}$. Non-profit safety organizations such as the Association for the Advancement of Medical Instrumentation (AAMI) and the ECRI Institute have proclaimed alarm management as a priority ${ }^{[4]}$. The ECRI Institute has identified many present-day challenges in alarm management ${ }^{[5]}$. The Joint Commission has reissued alarm management to improve the safety of clinical alarm systems as one of its National Patient Safety Goals for this year ${ }^{[6]}$.

Chopra and McMahon ${ }^{[7]}$ in a recent JAMA editorial stated “...what is needed is a complete reimagining of alarm systems from a patient-centric perspective”. We describe a new approach to alarm management that provides systematic redundancy to alarm surveillance that approaches fail-safe response to improve patient safety. The opportunity of a newly constructed children's hospital included plans for a specialized location where the digital output from every monitored patient is aggregated and displayed in a single location and staffed by a trained observer. We also outfitted each inpatient location with video surveillance and hands-free audio communication. The skilled observer is assigned to observe for abnormal signals providing an extra set of eyes and ears for alarm management. This alarm management strategy is unlike others that emphasize continuous improvement for single-point failure. We apply redundancy and believe it affordable and very promising.

\section{Remote alarm monitoring unit within a hospital}

At our freestanding, pediatric, tertiary care, 100-bed hospital, a room was created during the facility's recent construction to bring the alarms of these and other technologies for alarm management to one central location, the Clinical Logistics Center (CLC). The CLC was designed to receive the outputs of all the aforementioned technology from all patient locations via cable, phone line, or wifi signal. Medics with pediatric emergency medicine experience staff the CLC 24 hours a day in four-hour shifts. The medic's workstation comprises four monitors displaying monitoring data from all patient rooms, a desktop computer, a centralized patient alarm/intercom system, a hospital phone, and smartphone (see the figure). Physiologic variables including pulse, respiratory rate, oxygen saturation with plethysmograph, etc., are displayed on one of these monitors, and the EMR is displayed for access and easy viewing on a second monitor.

A third monitor displays to the paramedic in table format alerts from customizable software designed to detect real-time specific patient characteristics within the EMR (Epic Monitor, Epic Systems Corp., Verona, Wisconsin). For example, the combination of fever and an elevated white blood cell count in a patient with a central venous catheter alerts the paramedic to search the EMR for specific antibiotics to avoid a central-line-associated bloodstream infection. The patient's attending physician is readily identified via an electronic schedule located on the hospital's intranet and is then notified via text in the event that the appropriate antibiotics are not being received by the patient at risk for infection. Other conditions similarly tracked and managed include catheter-associated urinary tract infections and neonatal sepsis. The Pediatric Early Warning 
Scores (PEWS) ${ }^{[8]}$ entered by the nurse are also electronically tracked to assure proper escalation of monitoring and potentially higher level of care if needed.

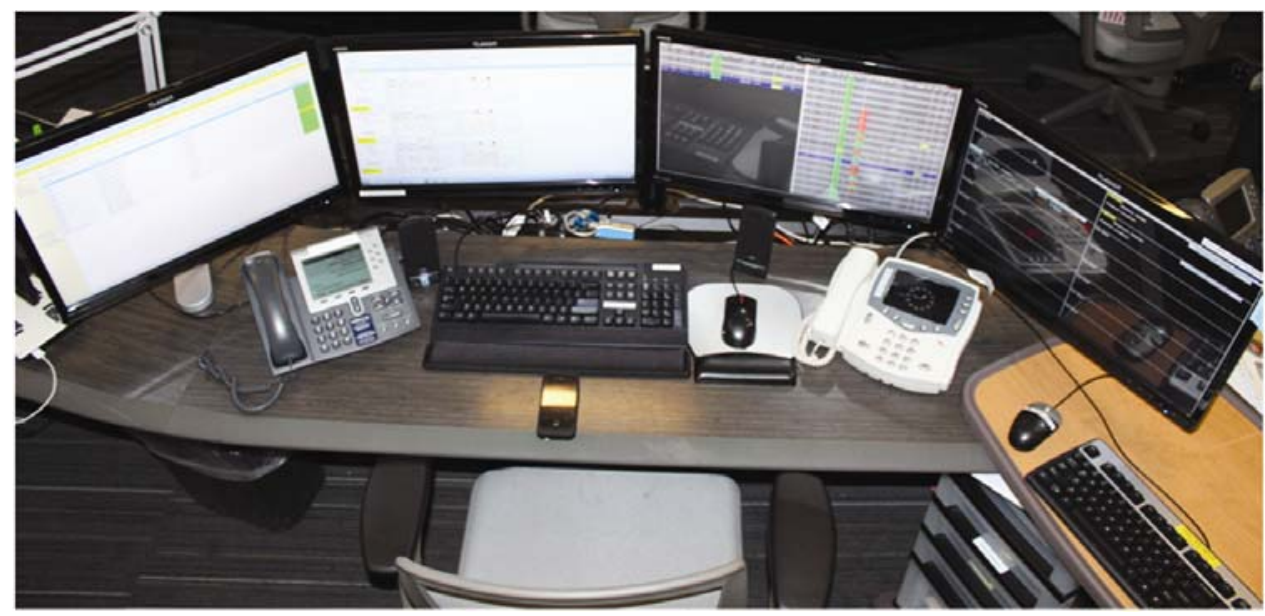

Figure. Medic workstation within the Clinical Logistics Center

A fourth monitor is used to view the location of all inpatients using an elopement/abduction alarm system (Totguard, Guard RFID Solutions, Inc., Delta, British Columbia). To ensure infant and child safety, a personal GPS locator is placed on the extremity of every patient so that his or her location is confirmed. The paramedic is alerted to alarms caused by the movement of a patient to an unwanted area such as a stairwell or elevators or if someone tampers with the locator. The software program used to communicate with bedside staff via text (Voalte Messenger, Voalte, Sarasota, Florida) can be accessed using the smartphone or desktop computer with display on one of the monitors. All of the alarms monitored by the paramedic are managed with protocols to encourage standard practice among the paramedics.

\section{Alarm management logistics/ processes}

Timely, direct communication of clinically relevant alarms from paramedic to bedside staff is critical to the appropriate management of patient alarms. Patient alarms are integrated with the bedside staff's smartphones via middleware (Emergin Orchestrator, Philips Healthcare, Andover, Massachusetts). Patient alarms not acknowledged by the bedside staff are escalated to the paramedic in the CLC after two minutes. The paramedic is not only cognizant of the response time of the bedside staff to an alarm, but he or she is also capable of assessing the quality of the alarm signal. For example, a pulse oximetry alarm of hypoxemia confirmed visually by the paramedic to have a good plethysmograph is communicated via smartphone text or voice call to the bedside nurse to ensure a more timely response. If the assigned nurse does not respond to the alarm notification by the paramedic in a timely manner, the paramedic escalates the alarm to another nurse located in the vicinity of the patient whose alarm is activated. The alarm is escalated to the charge nurse in a tiered response if necessary. When the paramedic detects a rapid clinical deterioration in the patient's condition, the paramedic has immediate audio and video contact with the patient's room and assesses the patient's appearance and presence of bedside staff. One notable incident was that of a child with epilepsy who had a seizure in the middle of the night that activated tachycardia and hypoxemia alarms, and the paramedic immediately established audio and video communication with the patient's room. In the absence of staff at the bedside during this true emergency, a rapid response alert was sent by the paramedic to the appropriate providers. The rapid response team arrived and delivered the anti-seizure medications within a few minutes of the start of the seizure. Rapid response alerts based solely on vital sign threshold values result in an exceedingly high number of false alerts and a waste of valuable resources, especially the rapid response team's responsibilities to their primary patients ${ }^{[9]}$. During a code blue alert activated by a bedside nurse for a life-threatening arrhythmia in a separate incident, the paramedic performed duties requested via the patient room intercom/centralized alarm system (Responder 5, Rauland-Borg of Florida, Altamonte Springs, Florida), such as calling the cardiologist on call, 
radiology technician for a stat chest radiograph, and respiratory therapist for a stat electrocardiogram, etc. Assisting the bedside staff with these communications allows all the bedside staff to remain at the patient's bedside assisting in the resuscitation. Direct visualization of patient rooms during GPS alarms also confirms the location and condition of the patient within the patient room to quickly detect true vs. false alarms. The table summarizes the scope of current and future functions of the CLC.

Table. Clinical Logistics Center current and future functions

\begin{tabular}{|c|c|}
\hline Current Functions & Examples \\
\hline Third-tier alarm escalation of physiologic monitors & High pulse, low $\mathrm{SpO}_{2}$ \\
\hline \multicolumn{2}{|l|}{ Remind bedside RN of overdue medications } \\
\hline Alert providers regarding user-defined clinical decision support & Reassess patient with rising severity of illness \\
\hline Oversee electronic watch list for clinical rules violations & Central line + fever + elevated WBC count \\
\hline Peruse medical records for required documentation & Handoffs, restraints \\
\hline \multicolumn{2}{|l|}{ Second tier for incoming phone calls regarding transfers, admits } \\
\hline Rapid response team alerts and logistical assistance & Alert attending physicians of clinical change \\
\hline Assist during code blue resuscitations & $\begin{array}{l}\text { Paging radiology technician, on call physicians to the patient's } \\
\text { bedside }\end{array}$ \\
\hline \multicolumn{2}{|l|}{ Future Functions } \\
\hline \multicolumn{2}{|l|}{ Assist with coordinating resources during high census } \\
\hline \multicolumn{2}{|l|}{ Scribe verbal orders into EMR during resuscitations } \\
\hline \multicolumn{2}{|l|}{ Communication center for interfacility transfers } \\
\hline \multicolumn{2}{|l|}{ Transport team dispatching and location tracking } \\
\hline Just-in-time coordination of operating rooms after hours & Assigning float personnel to ED, floors \\
\hline \multicolumn{2}{|l|}{ Interfacility telemedicine linkage } \\
\hline \multicolumn{2}{|l|}{ Additional protocol-based decision supports } \\
\hline \multicolumn{2}{|l|}{ Coordination of intrafacility patient transport } \\
\hline Physician priority line management & \\
\hline
\end{tabular}

\section{Discussion}

Alarm hazards top the ECRI Institute's top ten health technology hazards list again for $2014{ }^{[10]}$. One of the ECRI Institute's recommended goals for a comprehensive alarm management program is "optimizing alarm notification and response protocols so that the patient receives the appropriate care at the time it's needed". The CLC uses an innovative approach to alarm management utilizing today's technology to accomplish this goal. Confirming the quality of the alarm signal, immediately communicating true alarms to the bedside staff, and making direct audiovisual contact with the patient all contribute to this goal. The Joint Commission declared improving the safety of clinical alarm systems as a hospital national patient safety goal for this year ${ }^{[6]}$. The Joint Commission recommends identifying the most important alarm signals to manage as this year's focus for hospitals. The CLC's alarm prioritization and notification for the bedside staff go a long way toward meeting this recommendation. The bedside staff has worked with the CLC since its inception in improving its processes of alarm management to advance us toward the goal of alarms always enhancing patient safety, not detracting from it.

Limitations of the CLC are a result of its young age. Cultural acceptance by the bedside staff is slowly growing. Many staff members do not have experience with this innovative alarm management approach. Personal experience with the benefits to the patient is driving its cultural acceptance and growth. Continuous improvement to the processes with input from the bedside staff is integral to continued acceptance. Adjusting the alarm management protocols to decrease nuisance alerts to the bedside staff will reduce any additional alarm fatigue caused by the CLC. Some nurses request the paramedic to closely 
monitor individual patients during chemotherapy, electrolyte administration, or at the first signs of clinical deterioration. The maximum number of patient alarms one paramedic can optimally monitor and communicate about is unclear. Data collection, management, and analysis from all devices to determine outcomes such as response times are challenges that require further investment. Delivery of relevant information to the bedside staff using a single integrated display instead of the fragmented delivery with today's technology is needed to improve efficiency ${ }^{[11]}$. Demonstrating a quantifiable return on investment in technology and manpower is essential for widespread penetration of this innovative approach to alarm management.

\section{Conclusion}

Maintaining the priorities of alarms for the bedside staff and escalating true alarms in a timely manner is just the start of possible benefits of the CLC to patient safety. Imagination and innovation must continue to be applied to improve the safety of clinical alarms.

\section{References}

[1] Lawless ST. Crying wolf: false alarms in a pediatric intensive care unit. Crit Care Med. 1994 Jun; 22(6): 981-5. Available from: http://journals.lww.com/ccmjournal/Abstract/1994/06000/Crying_wolf_False_alarms_in_a_pediatric_intensive.17.aspx. PMid: 8205831. http://dx.doi.org/10.1097/00003246-199406000-00017

[2] De Man FR, Greuters S, Boer C, Veerman DP, Loer SA. Intra-operative monitoring - many alarms with minor impact. Anaesthesia. 2013; 68: 804-10. http://dx.doi.org/10.1111/anae.12289

[3] Joint Commission Sentinel Event Alert Issue 50: Medical device alarm safety in hospitals [cited 2014 Feb 4]. Available from: http://www.jointcommission.org/sea_issue_50/

[4] McKinney M. Lives at risk. Hospitals face hurdles addressing alarm fatigue. Modern Healthcare. 2013; 43(15): 14-15. Available from: http://web.a.ebscohost.com/ehost/external?sid=0ad259a0-e620-4e93-856b-f208a2dacccf\%40sessionmgr4004\&vid=3\&hid=420 7

[5] Addis LM, Cadet VN, Graham KC. Sound the alarm. Patient Safety \& Quality Healthcare. 2014; 11(3): 40-4. Available from: http://www.psqh.com/may-june-2014/2255-sound-the-alarm

[6] Joint Commission. Hospital: 2014 national patient safety goals [cited 2014 Feb 4]. Available from: http://www.jointcommission.org/hap_2014_npsgs/ .

[7] Chopra V, McMahon LF Jr. Redesigning hospital alarms for patient safety. Alarmed and potentially dangerous. JAMA. 2014 Mar 26; 311(12): 1199-1200. http://dx/doi.org/10.1001/jama.2014.710

[8] Akre M, Finkelstein M, Erickson M, et al. Sensitivity of the Pediatric Early Warning Score to identify patient deterioration. Pediatrics. 2010; 125: e763-769. http://dx.doi.org/10.1542/peds.2009-0338

[9] Fagan K, Sabel A, Mehler PS, MacKenzie TD. Vital sign abnormalities, rapid response, and adverse outcomes in hospitalized patients. Am J Med Qual. 2012 Nov-Dec; 27(6): 480-6. http://dx.doi.org/10.1177/1062860611436127

[10] ECRI Institute. Top ten health technology hazards for 2014 [cited 2014 Feb 11]. Available from: https://www.ecri.org/Forms/Documents/2014_Top_10_Hazards_Executive_Brief.pdf

[11] Koch SH, Staggers N, Weir C, Agutter J, Liu D, Westenskow DR. Integrated information displays for nurses: field observations, display design, and display evaluation. Proceedings of the Human Factors and Ergonomics Society Annual Meeting. 2010; 54: 932-6. http://dx.doi.org/10.1177/154193121005401227 